\title{
MODEL FOR ORGANIZATION CARGO TRANSPORTATION AT RESOURCE RESTRICTIONS
}

\author{
Levon A. Beklaryan ${ }^{1}$, Nerses K. Khachatryan ${ }^{2}$, \\ Andranik S. Akopov ${ }^{3}$ \\ ${ }^{1}$ Central Economics and Mathematics Institute \\ of Russian Academy of Sciences \\ Nakhimovsky Prospect, 47, 117418 - Moscow, RUSSIA \\ ${ }^{2}$ Plekhanov Russian University of Economics \\ Stremyanny lane, 36, 117997 - Moscow, RUSSIA \\ ${ }^{3}$ Plekhanov Russian University of Economics \\ Stremyanny lane, 36, 117997 - Moscow, RUSSIA
}

\begin{abstract}
The model for organizing railway transportation on a long stretch of road between two node stations connected by a large number of intermediate stations is investigated. It allows to predict dynamics of load of the stations and flows arising in transport network at the set procedure of the movement of freight traffic. Such model is given by a system of differential equations with discontinuous right parts and satisfying the conditions setting resource restrictions. The numerical realization of this system is carried out. Depending on the ratio of the intensity of sending goods to the initial node station and the intensity of the distribution of goods from the final node station, three types of solutions of a given system are identified.
\end{abstract}

\section{AMS Subject Classification: $34 \mathrm{~A} 36$}

Key Words: organization of cargo transportation, modeling of traffic flows, intensity, differential equations, numerical realization

\section{Introduction}

The correct organization of the movement in transport network assumes the Received: June 13, 2019 (c) 2019 Academic Publications

${ }^{\S}$ Correspondence author 
use of control systems. Their algorithms are based on mathematical models, one of the main functions of which is modeling of traffic flows. A large number of publications is devoted to mathematical modeling of traffic flows. The works [1], [2], [3] describe "analog models" in which the movement of the vehicle is similar to any physical flow (hydro - and gas - dynamic models). There is a class of models in which solved the problem of optimization of transportation routes, generating optimal network configuration, etc (see [4], [5], [6], [7]). One of the approaches to modeling and research of traffic flows is based on the theory of competitive non-coalition equilibrium (see [8], [9], [10], [11]). It allows to describe a fairly adequate mechanism for the functioning of road networks. We also note the approach associated with the use of simulation and cellular automata described in [12], [13], [14], [15]. A number of publications are devoted to the modeling of rail transportation and flows arising in their process (see [16], [17], [18], [19], [20], [21], [22]). In particular, in works [19], [20], [21], [22] a model of organization of rail freight between two node stations connected by a railway line which contains a certain number of intermediate stations is investigated. It is assumed that between arbitrary stations there is interexchange railway track, where part of the cargo can be temporarily stored. Such model allows to predict dynamics of load of the stations and flows arising in transport network at the set procedure of the movement of freight traffic. The procedure of the movement of freight traffic uses two technologies uniform for all stations. The first technology is based on interaction of the neighboring stations and is formed by a certain rule. According to this rule, each of stations has to take the cargo from the previous station if the quantity of the involved roads on it are less than at the previous station, and to send on the next station if the quantity of the involved roads on it are more than at the next station. In this case, both the intensity of reception, and intensity of shipment cargo is proportional to the difference of numbers of the involved roads at the neighboring stations. The second technology uses technical capabilities of the station and is based on interaction of the station with the neighboring railway tracks. In these models it was supposed that capacities of railway tracks are unlimited. The modification of model considering limitation of railway tracks is given in this article.

\section{Description of model}

The movement of goods is carried out in one direction. The traffic flow diagram is shown in Fig. 1. 


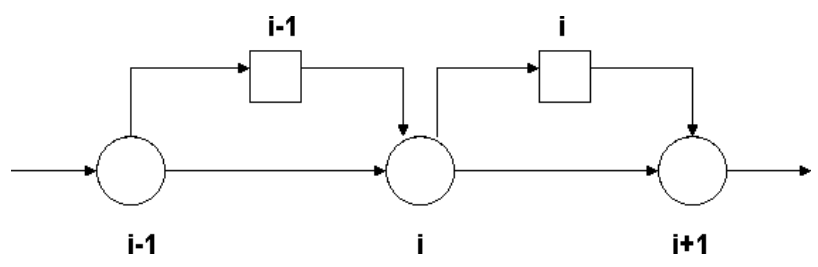

Fig. 1. The traffic flow diagram

In this figure, the circles indicate the stations, and the squares indicate the railway tracks. As can be seen from the figure, cargo can arrive at an arbitrary intermediate station both from the previous station and from the railway track, and sent from it either to the next station or to the railway track.

Let the number of intermediate stations be equal to $m$. Denoting by 0 and $m+1$, respectively the numbers of the initial and final nodal stations, we obtain the following set of station numbers: $\{0,1, . ., m, m+1\}$.

Let us move on to the numbering of the railway tracks. The railway track located between stations with numbers $i$ and $i+1$ is denoted by number $i$. Thus, we obtain the following set of railway track: $\{0,1, . ., m\}$. Each station and each railway track at any time are characterized by the number of involved roads. Denote by $z_{i}(t), i=0,1, \ldots, m+1$ and $y_{i}(t), i=0,1, \ldots, m$, respectively, the number of roads involved at $i$ - th station and $i$ - th railway track at time $t$. The maximum number of involved roads at the stations, at which the mode of increasing the number of roads at the expense of goods from the railway track, is functioning, we denote by $\Delta$. If the number of involved roads exceeds this value, part of the cargo is temporarily sent to the railway track. It is assumed that the number of roads on all railway tracks is equal to $V$.

The organization of cargo traffic is carried out using two technologies.

The first technology is based on the interaction procedure of neighboring stations. Each station with number $1 \leq i \leq m$ must take the cargo from the previous station with an intensity equal to $\alpha\left(z_{i-1}-z_{i}\right)$ if $z_{i-1}>z_{i}$ and send the cargo to the next station with an intensity equal $\alpha\left(z_{i}-z_{i+1}\right)$ if $z_{i}>z_{i+1}$. If the first condition is violated, the station with number $i$ sends the cargo to the railway track with number $i$ with intensity $\alpha\left(z_{i}-z_{i-1}\right)$ if $y_{i}<V$, and if the second one is violated, it receives the cargo from the railway track with number $i-1$ with intensity $\alpha\left(z_{i+1}-z_{i}\right)$ if $y_{i-1}>0$. Initial node station $(i=0)$ takes a cargo with intensity $\psi_{1}(t) \geq 0$ and sends it to the next station with intensity $\alpha\left(z_{0}-z_{1}\right)$ if $z_{0}>z_{1}$. Otherwise, the initial node station additionally takes the cargo with the intensity $\alpha\left(z_{1}-z_{0}\right)$. Final node station $(i=m+1)$ accepts the cargo from the previous station with intensity $\alpha\left(z_{m}-z_{m+1}\right)$ if $z_{m}>z_{m+1}$ and 
distributes it with intensity $\psi_{2}(t) \geq 0$. If $z_{m}<z_{m+1}$, then the final station additionally distributes the cargo with intensity $\alpha\left(z_{m+1}-z_{m}\right)$.

The second technology is designed to use the infrastructure capabilities of the stations and to ensure uninterrupted movement of cargo. It is based on the procedure of interaction of the station with neighboring railway tracks located on opposite sides of it. The second technology for all stations, except the initial one, allows us to increase the number of involved roads (if it does not exceed $\Delta$ ) and to reduce it (if it exceeds $\Delta$ ). The function $\varphi($.$) , setting the speed of$ change of number of involved roads within this technology has the following properties: on a half-line $(-\infty, 0)$ it is identically equal to zero, on an interval $\left(0, x_{\text {opt }}\right)$ is increasing, in a point $x_{\text {opt }}$ accepts the maximum value, on a half-line $\left(x_{\text {opt }},+\infty\right)$ is decreasing, in a point $\Delta$ accepts zero value, and on a half-line $(\Delta,+\infty)$ is linear. Thus, within the second technology, the station with the number $i=1,2, \ldots, m$ accepts cargo with $(i-1)$ - th railway track with intensity $\varphi\left(x_{i}\right)$ if $x_{i}<\Delta$ and $y_{i-1}>0$ and sends the cargo to $i$ - th railway track with intensity $\varphi\left(x_{i}\right)$ if $x_{i}>\Delta$ and $y_{i}<V$. Final node station $(i=m+1)$ within the second technology accepts cargo with $m$ - th railway track with intensity $\varphi\left(x_{m+1}\right)$ if $x_{m+1}<\Delta$ and $y_{m}>0$ and additionally distributes the cargo with intensity $\varphi\left(x_{m+1}\right)$ if $x_{m+1}>\Delta$.

For the initial node station $(i=0)$ the second technology is used only for unloading. The function $\varphi_{0}($.$) , setting the speed of change of the number of$ involved roads at this station within this technology, has the following properties: on a half-line $(-\infty, \Delta)$ it is identically equal to zero, and on a half-line $(\Delta,+\infty)$ it is linearly decreasing. Thus, the initial node station $(i=0)$ sends the cargo to 0 - th railway track with intensity $\varphi\left(x_{0}\right)$ if $x_{0}>\Delta$ and $y_{0}<V$.

Thus, the dynamics of numbers of the involved roads at stations is set by the system of the differential equations

$$
\begin{aligned}
\dot{z}_{0}(t)= & \psi_{1}(t)-\alpha\left(z_{0}-z_{1}\right) \\
& +\varphi_{0}\left(z_{0}\right) \operatorname{sign}\left(z_{0}-\Delta\right) \operatorname{sign}\left(V-y_{0}\right), \quad t \in[0,+\infty), \\
\dot{z}_{i}(t)= & \alpha\left(z_{i-1}-z_{i}\right) \operatorname{sign}\left(z_{i-1}-z_{i}\right) \\
& +\alpha\left(z_{i-1}-z_{i}\right) \operatorname{sign}\left(z_{i}-z_{i-1}\right) \operatorname{sign}\left(V-y_{i}\right) \\
& -\alpha\left(z_{i}-z_{i+1}\right) \operatorname{sign}\left(z_{i}-z_{i+1}\right) \\
& +\alpha\left(z_{i}-z_{i+1}\right) \operatorname{sign}\left(z_{i+1}-z_{i}\right) \operatorname{sign}\left(y_{i-1}\right) \\
& +\varphi\left(z_{i}\right) \operatorname{sign}\left(\Delta-z_{i}\right) \operatorname{sign}\left(y_{i-1}\right) \\
& +\varphi\left(z_{i}\right) \operatorname{sign}\left(z_{i}-\Delta\right) \operatorname{sign}\left(V-y_{i}\right), \\
& \quad i=1,2 \ldots, m, \quad t \in[0,+\infty), \\
\dot{z}_{m+1}(t)= & \alpha\left(z_{m}-z_{m+1}\right)-\psi_{2}(t)
\end{aligned}
$$




$$
\begin{aligned}
& +\varphi\left(z_{m+1}\right) \operatorname{sign}\left(\Delta-z_{m}\right) \operatorname{sign}\left(y_{m}\right) \\
& +\varphi\left(z_{m+1}\right) \operatorname{sign}\left(z_{m}-\Delta\right) \operatorname{sign}\left(V-z_{m}\right), \quad t \in[0,+\infty) .
\end{aligned}
$$

The function $\operatorname{sign}($.$) involved in equations (1)-(3)$ is defined as follows:

$$
\operatorname{sign}(t)= \begin{cases}1, & \text { if } t>0 \\ 0, & \text { if } t \leq 0\end{cases}
$$

We investigate the dynamics of capacities of railway track. Determine with what intensity cargo come on the railway tracks and with what intensity leave them. Within the first technology, on a stage with number $1 \leq i \leq m-1$ cargo arrives from the station with number $i$ with intensity $\alpha\left(z_{i}-z_{i-1}\right)$ if $z_{i}>z_{i-1}$ and $y_{i}<V$ and goes to the station with number $i+1$ with intensity $\alpha\left(z_{i+2}-z_{i+1}\right)$ if $z_{i+2}>z_{i+1}$ and $y_{i}>0$. On an initial railway track $(i=0)$ within the first technology cargo does not arrive, goes from it to the station with number 1 with intensity $\alpha\left(z_{2}-z_{1}\right)$ if $z_{2}>z_{1}$ and $y_{0}>0$. At last, on a final railway track $(i=m)$ within the same technology cargo arrives from the station with number $i=m$ with intensity $\alpha\left(z_{m}-z_{m-1}\right)$ if $z_{m}>z_{m-1}$ and $y_{m}<V$. The cargo is not sent from the final railway track within this technology.

Within the second technology, on a railway track with number $0 \leq i \leq m$ cargo arrives from the station with number $i$ with intensity $-\varphi\left(z_{i}\right)$ if the number of the involved roads at the station with number $i$ exceeds value $\Delta$ and $y_{i}<V$ and goes to the station with number $i+1$ with intensity $\varphi\left(z_{i+1}\right)$ if the number of the involved roads at the station with number $i+1$ is less than value $\Delta$ and $y_{i}>0$ (the station with number $i+1$ accepts cargo from a railway track).

Thus, the dynamics of the number of the involved roads on a railway track is described by the following system of differential equations

$$
\begin{aligned}
\dot{y}_{0}(t)= & -\alpha\left(z_{2}-z_{1}\right) \operatorname{sign}\left(z_{2}-z_{1}\right) \operatorname{sign}\left(y_{0}\right) \\
& -\varphi\left(z_{0}\right) \operatorname{sign}\left(z_{0}-\Delta\right) \operatorname{sign}\left(V-y_{0}\right) \\
& -\varphi\left(z_{1}\right) \operatorname{sign}\left(\Delta-z_{1}\right) \operatorname{sign}\left(y_{0}\right), \quad t \in[0,+\infty), \\
\dot{y}_{i}(t)= & \alpha\left(z_{i}-z_{i-1}\right) \operatorname{sign}\left(z_{i}-z_{i-1}\right) \operatorname{sign}\left(V-y_{i}\right) \\
& -\alpha\left(z_{i+2}-z_{i+1}\right) \operatorname{sign}\left(z_{i+2}-z_{i+1}\right) \operatorname{sign}\left(y_{i}\right) \\
& -\varphi\left(z_{i}\right) \operatorname{sign}\left(z_{i}-\Delta\right) \operatorname{sign}\left(V-y_{i}\right) \\
& -\varphi\left(z_{i+1}\right) \operatorname{sign}\left(\Delta-z_{i+1}\right) \operatorname{sign}\left(y_{i}\right) \\
& \quad i=1,2 \ldots, m-1, \quad t \in[0,+\infty) \\
\dot{y}_{m}(t)= & \alpha\left(z_{m}-z_{m-1}\right) \operatorname{sign}\left(z_{m}-z_{m-1}\right) \operatorname{sign}\left(V-y_{m}\right) \\
& -\varphi\left(z_{m}\right) \operatorname{sign}\left(z_{m}-\Delta\right) \operatorname{sign}\left(V-y_{m}\right)
\end{aligned}
$$




$$
-\varphi\left(z_{m+1}\right) \operatorname{sign}\left(\Delta-z_{m+1}\right) \operatorname{sign}\left(y_{m}\right), \quad t \in[0,+\infty) .
$$

Besides, the inequalities have to be carried out:

$$
0 \leq y_{i}(t) \leq V, \quad i=0,1, \ldots, m, \quad t \in[0,+\infty) .
$$

These inequalities are imposed by restriction for number of the involved roads on railway tracks.

Thus, the model for organization cargo transportation investigated by us is described by a system (1)-(7).

The analytical research of solutions of system (1) - (7) extremely difficult, as the right parts of the differential equations (1)-(6) are discontinuous functions. In this regard, the system (1) - (7) was investigated numerically. We present the results of a numerical research.

\section{Results of numerical experiments}

For periodic functions $\psi_{1}(t)=d+\gamma \cos (\omega t), d \geq \gamma>0, \quad \psi_{2}(t)=\lambda \psi_{1}(t), \lambda>0$ and functions $\varphi($.$) and \varphi_{0}($.$) defined as follows$

$$
\begin{gathered}
\varphi(x)=\left\{\begin{array}{l}
0, \text { if } x<0 \\
a x(\Delta-x), a>0 \text { if } x \in[0, \Delta] \\
-c(x-\Delta), c>0 \text { if } x \in(\Delta,+\infty),
\end{array}\right. \\
\varphi_{0}(x)=\left\{\begin{array}{l}
0, \text { if } x \leq \Delta \\
-c_{0}(x-\Delta), c_{0}>0 \text { if } x \in(\Delta,+\infty),
\end{array}\right.
\end{gathered}
$$

numerical solutions of a system (1) - (7) were received.

Depending on parameter $\lambda$, participating in definition of function $\psi_{2}($.$) , we$ receive three types of solutions of system $(1)-(7)$. They define three types of dynamics of the involved roads at stations and railway tracks. Before we pass to detailed studying of each of three types of solutions of system (1) - (7), we will note the property of solutions of this system characteristic of all three types. Numerous experiments showed that there is a point in time $t_{0}$ such that the condition

$$
z_{0}(t)>z_{1}(t)>\ldots>z_{m}(t)>z_{m+1}(t), \quad t \in\left[t_{0},+\infty\right)
$$

is satisfied.

The first type of the solution of a system (1)-(7) $(0<\lambda<1)$ : 
This type of solution is characterized by existence of point in time $t_{1}>t_{0}$ such that for all $t \geq t_{1}$ and any initial values, the components of the solution of a system $(1)-(7)$ satisfy the following conditions:

$$
z_{i}(t)>\Delta, i=0,1, \ldots, m+1, \quad y_{i}(t)=V, \quad i=0,1, \ldots, m .
$$

Let us note that the value of $t_{1}$ increases both at increase in value of $V$, and at increase in value of $\lambda$. This type of solutions can be seen in Fig. 2. The dynamics of the number of involved roads at the stations (the number of stations is equal to 10) is shown above, and the dynamics of the number of involved roads at the railway tracks (the number of railway tracks, respectively, is equal to 9) is shown below. The value of $\Delta$ is equal to 10 and the value of $V$ is equal to 15.
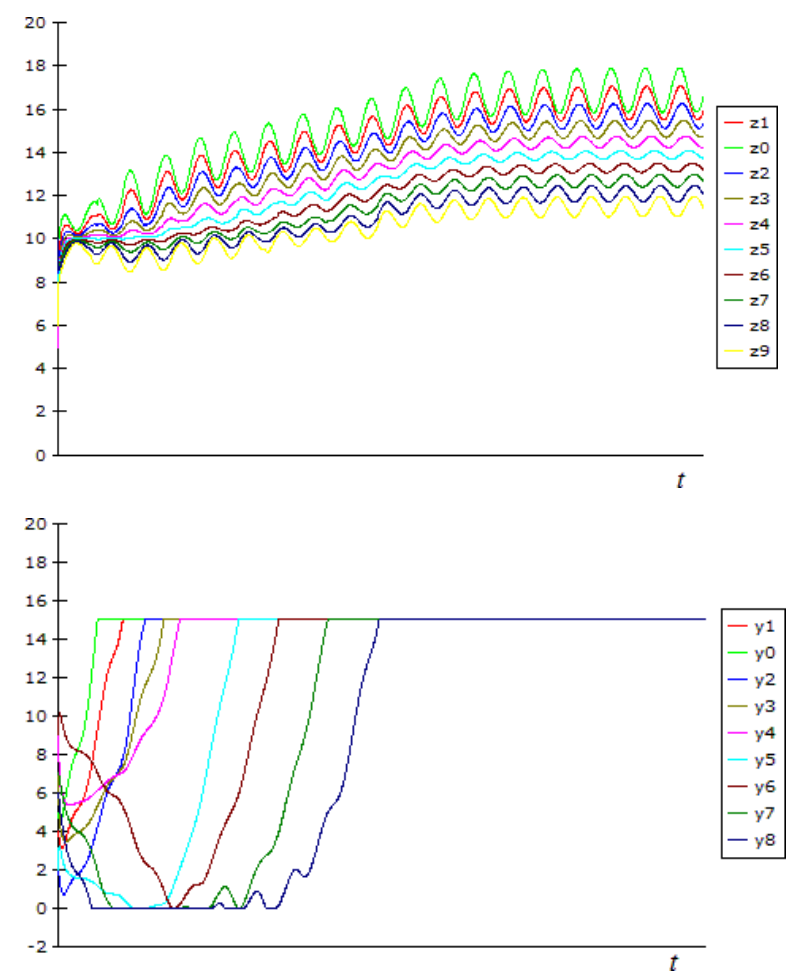

Fig. 2. The first type of solution of system $(1)-(7)$

As shown in Fig. 2, starting from a certain point in time, the number of involved roads at all stations in turn, starting from the initial, exceeds the value of $\Delta$ and parallel with it, also in turn, the number of involved roads on 
all railway tracks since the initial reaches the maximum value $V$. After that, according to the rules of organization of cargo transportation, the process of organization of cargo transportation is carried out only within the first technology without involving railway tracks (since the number of involved roads at each next station is less than at the previous one).

The second type of solution of system $(1)-(7)(\lambda=1)$ :

This type of solution is characterized by existence of point in time $t_{2}$ such that for all $t \geq t_{2}$ the components of the solution of a system (1) - (7) satisfy one of the following conditions:

$$
\begin{gathered}
z_{i}(t)>\Delta, i=0, \ldots, \bar{m}, z_{i}(t)<\Delta, i=\overline{\bar{m}}, \ldots, m+1, \\
y_{i}(t)=V, \quad i=0, \ldots, \bar{m}, y_{i}(t)=0, i=\overline{\bar{m}}, \ldots, m
\end{gathered}
$$

where

$$
0 \leq \bar{m}<\overline{\bar{m}} \leq m+1
$$

or

$$
z_{i}(t)<\Delta, i=0, \ldots, m+1, \quad y_{i}(t)=0, i=0, \ldots, m
$$

or

$$
z_{i}(t)>\Delta, i=0, \ldots, m+1, \quad y_{i}(t)=V, i=0, \ldots, m .
$$

Feasibility of this or that condition $((8)-(9),(10)$ or $(11))$, and also values of numbers $\bar{m}$ and $\overline{\bar{m}}$ depend on initial values of system $(1)-(7)$ and on the value of $V$. Independently of the value of $V$, the condition (10) is satisfied for small initial values of the system (1) - (7) (Fig. 4), the condition (11) - at large (Fig. 5), and conditions (8) - (9) - at intermediate (Fig. 3).

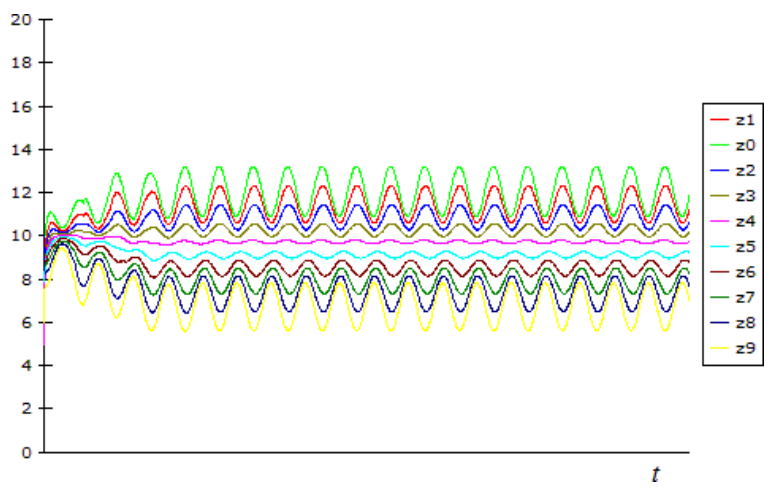




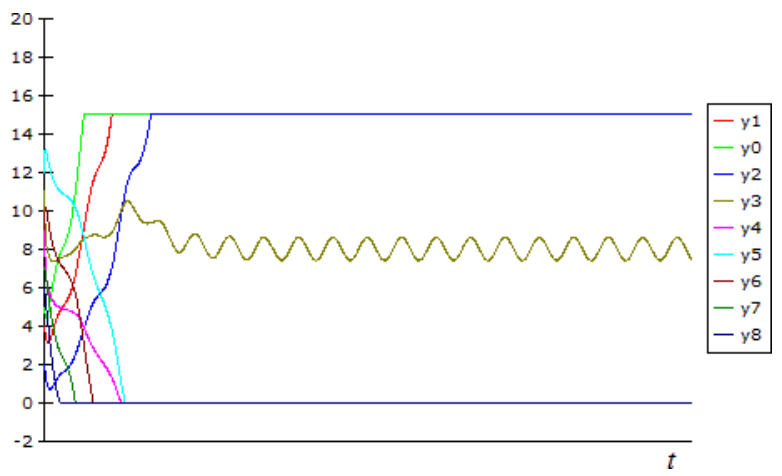

Fig. 3. The second type of solution of the system $(1)-(7)$ (case 1$)$
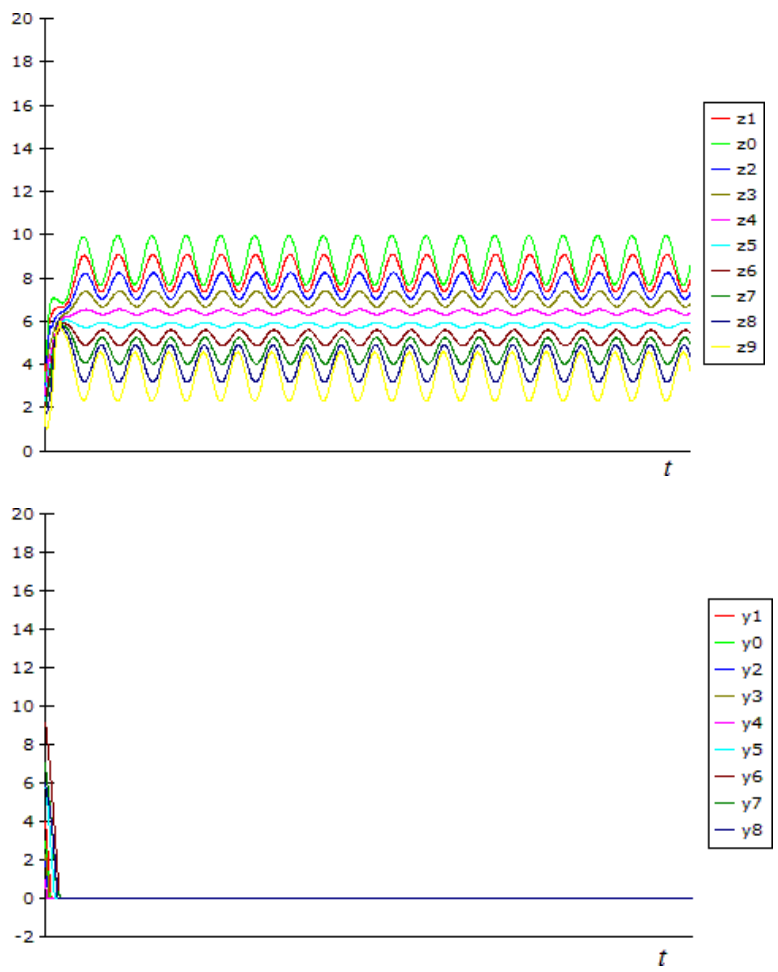

Fig. 4. The second type of solution of the system (1) - (7) (case 2) 

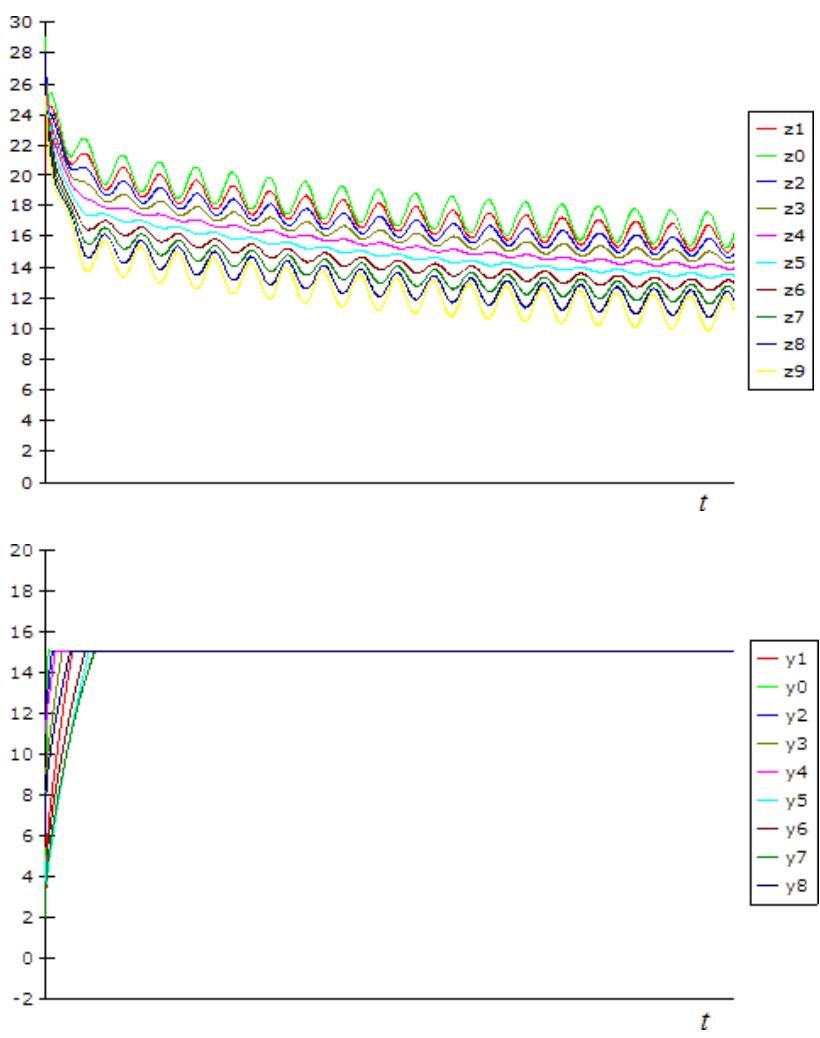

Fig. 5. The second type of solution of the system (1) - (7) (case 3)

The third type of solutions of the system (1)-(7) $(\lambda>1)$ :

This type of solution is characterized by existence of point in time $t_{3}$ such that for all $t \geq t_{3}$ and any initial values the components $y_{i}($.$) of solutions of the$ system (1) - (7) satisfy the following conditions:

$$
y_{i}(t)=0, i=0, \ldots, m,
$$

and components $z_{i}($.$) infinitely decrease (Fig. 6). Let us note that the value of$ $t_{3}$ increases both at increase in value of $V$, and at reduction of value $\lambda$. 

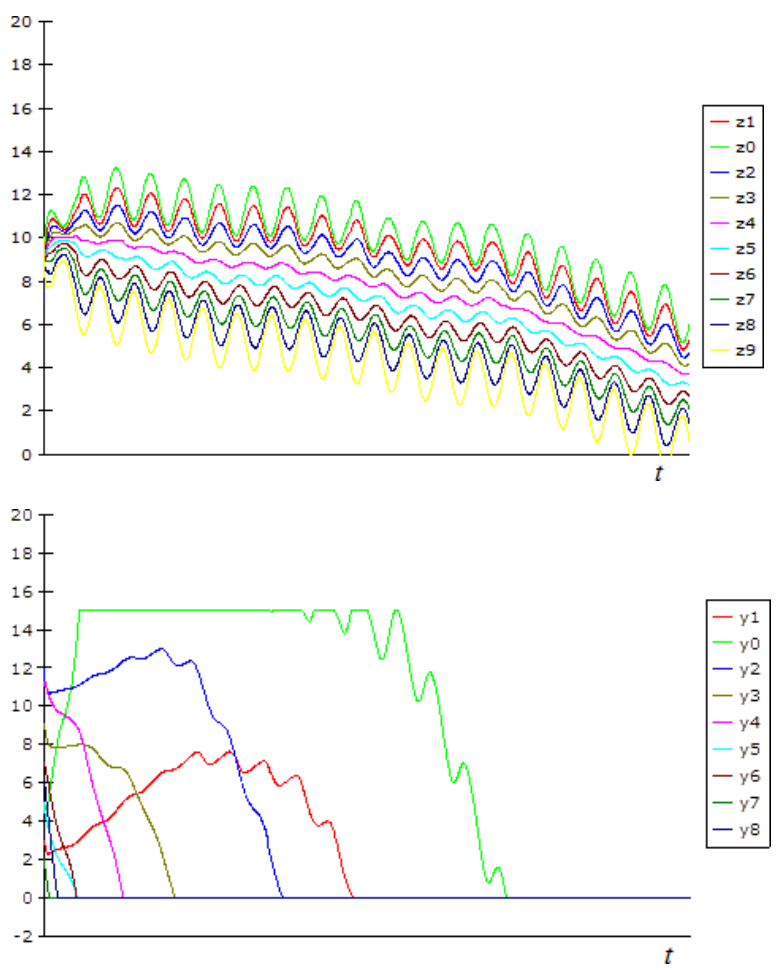

Fig. 6. The third type of solutions of the system $(1)-(7)$

Let us analyze the received results for each of three types of solutions of system $(1)-(7)$. The first type of solutions defines dynamics of number of the involved roads at stations and railway tracks in case the intensity of distribution of goods from the final node station is less than intensity of supply of goods on the initial node station. In this case over time there is a full filling of railway tracks, stations become strongly loaded and, as a result, the second technology is disconnected. The further movement of cargo flow is carried out only within the first technology. However, if the difference in intensities of supply of goods at the initial node station and distribution of goods with the final node station is small, it is possible to state that throughout all horizon of planning of transportations the second technology will remain involved.

The second type of solutions determines the dynamics of the number of involved roads in the stations and railway tracks in the case where the intensity of distribution of goods from the final node station is equal to the intensity of supply of goods to the initial node station. Recall that in this case, depending on the load of the stations at the initial time, there are three types of this 
dynamics. If at the initial moment of time the stations are involved slightly, then over time the stocks of goods on all railway tracks come to an end, the stations are loaded to a level below the optimum and it also leads to the shutdown of the second technology. Let us note that shutdown of the second technology after freeing the railway tracks is more preferable than shutdown after their overflow, since in the latter case it is necessary to connect additional resources for unloading cargo from the railway tracks. Opposite degree of fullness of the stations in the initial moment of time (critical overflow) in practice is extremely unlikely. Finally, at the most probable average degree of fullness of stations at the initial time, the most optimal station loading occurs, corresponding to the most efficient use of the stations infrastructure capabilities. At the same time, there is an overflow of the railway tracks close to the initial node station and the freeing of the railway tracks close to the final node station.

The third type of solutions determines the dynamics of the number of involved roads in the stations and railway tracks in the case where the intensity of distribution of goods from the final node station exceeds the intensity of supply of goods to the initial node station. In this case, over time, railway tracks are released, the second technology is turned off, and the load of stations is reduced to its complete inactivity. However, if the difference in the intensity of distribution of goods from the final node station and supply of goods to the initial node station is small, it is possible to state that throughout all horizon of planning of transportations the second technology will remain involved.

\section{Conclusion}

The model for organizing railway transportation between two node stations at limitation of capacities of the railway tracks located between stations is investigated. The movement of cargo is carried out by means of the set technologies describing interaction of the neighboring stations with each other and with railway tracks. Such a model is given by a system of differential equations with discontinuous right parts and satisfying the conditions that determine the boundedness of the railway tracks. Finding of the analytical solution of this system is extremely difficult, which leads to the need for its numerical realization. It is possible to describe the dynamics of number of the involved roads at stations and railway tracks depending on the ratio of the intensity of supply of goods to the initial node station and the intensity of distribution of goods from the final node station. 


\section{References}

[1] H. Inose, T. Hamada, Road Traffic Control, University of Tokyo Press, Tokyo (1975).

[2] A.B. Sukhinova, M.A. Trapeznikova, B.N. Chetvertushkin, N.G. Chubarova, Two-dimensional macroscopic model of traffic flows, Mathematical Models and Computer Simulations, 21, No 2 (2009), 118-126 (in Russian).

[3] Ya.A. Kholodov, A.S. Kholodov, A.V. Gasnikov, I.I. Morozov, V.N. Tarasov, Modeling of traffic flows-Topical problems and ways to solve them, Proc. of MIPT, 2, No 4 (2010), 152-162 (in Russian).

[4] V.G. Galaburda, Improving Transportation Technologies and an Increase in Rail Capacity, MIIT, Moscow (1983) (in Russian).

[5] V.G. Galaburda, Optimal Planning of Cargo Flows, Transport, Moscow (1985) (in Russian).

[6] O.I. Aven, S.E. Lovetsky, G.E. Moiseenko, Optimization of Transport Flows, Nauka, Moscow (1985) (in Russian).

[7] E.M. Vasilieva, R.V. Igudin, V.N. Livshits, Optimization of Planning and Management of Transport Systems, Transport, Moscow (1987) (in Russian).

[8] V.I. Shvetsov, Algorithms for distributing traffic flows, Automation and Remote Control, 70, No 10 (2009), 1728-1736.

[9] T. Leventhal, G.L. Nemhauser, L. Trotter, A column generation algorithm for optimal traffic assignment, Transportation Science, 7, No 2 (1973), 168-176.

[10] H.K. Lo, A. Chen, Traffic equlibrium problem with route-specific costs: formulation and algorithms, Transportation Research: Part B, 34, No 6 (2000), 493-513.

[11] H. Bar-Gera, Origin-based algorithm for the traffic assignment problem, Transportation Science, 36, No 4 (2002), 398-417.

[12] T. Yamada, B.F. Russ, J. Castro, E. Taniguchi, Designing multimodal freight transport networks: A heuristic approach and applications, Transportation Science, 43, No 2 (2009), 129-143. 
[13] M. Cremer, J. Ludwig, A fast simulation model for traffic flow on the basis of Boolean operations, Mathematics and Computers in Simulation, 28 (1986), 297-303.

[14] M.L. Blank, Exact analysis of dynamical systems arising in models of traffic flow, Russian Mathematical Surveys, 55, No 3 (2000), 562-563.

[15] A.P. Buslaev, A.V. Gasnikov, M.V. Yashina, Selected mathematical problems of traffic flow theory, International J. of Computer Mathematics, 89, No 3 (2012), 409-432.

[16] A.O. Rubtsov, A.S. Tarasov, Modeling of railway transportation in Russia, Proc. of the Institute for Systems Analysis of the Russian Academy of Sciences, 46 (2009), 274-278 (in Russian).

[17] D.N. Gainanov, A.V. Konygin, V.A. Rasskazova, Modelling railway freight traffic using the methods of graph theory and combinatorial optimization, Automation and Remote Control, 77, No 11 (2016), 1928-1943.

[18] D.Yu. Levin, Modeling of transportation processes, World of Transport and Transportation, 8, No 5 (2010), 48-55 (in Russian).

[19] L.A. Beklaryan, N.K. Khachatryan, Traveling wave type solutions in dynamic transport models, Functional Differential Equations, 13, No 12 (2006), 125-155.

[20] L.A. Beklaryan, N.K. Khachatryan, On one class of dynamic transportation models, Computational Mathematics and Mathematical Physics, 53, No 10 (2013), 1466-1482.

[21] N.K. Khachatryan, A.S. Akopov, Model for organizing cargo transportation with an initial station of departure and a final station of cargo distribution, Business Informatics, 39 (2017), 25-35.

[22] N.K, Khachatryan, A.S. Akopov, F.A. Belousov, About quasi-solutions of traveling wave type in models for organizing cargo transportation, Business Informatics, 43 (2018), 61-70. 